\title{
A Model for Stability Study of PFC Power Supplies
}

\author{
Grace Chu, Chi K. Tse and Siu Chung Wong \\ Department of Electronic and Information Engineering, Hong Kong Polytechnic University, Hong Kong
}

\begin{abstract}
Typically a power-factor-correction power supply consists of two stages, one responsible for power factor correction and the other for voltage regulation. In this paper, we propose to use a constant power sink to represent the voltage regulating stage, resulting in an analytically tractable model that is able to predict the period-doubled oscillations at line frequency which are not detectable by other conventional models. Using a generalized averaging approach, we investigate the lowfrequency dynamics and derive closed-form stability conditions which accurately locate the stability boundaries on selected parameter planes. The model can be conveniently used to evaluate the performance of power-factor-correction power supplies, such as harmonic distortion and power factor. Experimental results are presented to verify the model.
\end{abstract}

\section{INTRODUCTION}

Line frequency oscillation was first reported experimentally and by computer simulations in [1]. Although it has been recognized as a form of "period-doubling" phenomenon, the underlying mechanism can in fact be explained in terms of nonlinear forced oscillation, in which the nonlinearity of the system admits a unity loop gain at half the frequency of the forcing function (rectified line input) that causes oscillation at the line frequency, as analyzed thoroughly in [2] using a generalized averaging technique. However, the PFC boost converter has been assumed to be terminated by a resistive load. This assumption has been found causing significant discrepancies in the stability information produced [3]. In practice, the PFC boost converter is always terminated by a downstream $\mathrm{dc} / \mathrm{dc}$ converter with tightly regulated output voltage. The difference in stability information between resistor terminated PFC boost converter and the actual two-stage PFC power supply has been reported in [3]. In this paper, we extend the work of Wong et al. [2]. We re-examine the actual two-stage PFC power supply and propose a simple model for the downstream $\mathrm{dc} / \mathrm{dc}$ converter. Essentially, we model the tightly regulated downstream dc/dc converter as constant power sink. With this model, closed-form stability conditions can be derived and compared between the resistor terminated PFC converter and the proposed model. Experimental measurements will be presented to verify the analytical results. Furthermore, some design guidelines will be provided to illustrate possible applications.

\section{Modeling with Constant Power Sink}

A typical two-stage PFC power supply consists of a PFC pre-regulator which is cascaded by a downstream $\mathrm{dc} / \mathrm{dc}$ converter, as depicted in Fig 1 (a). The complete system is of relatively high order and analysis is thus rather complicated. Regardless of the fluctuation in its input voltage (i.e., output

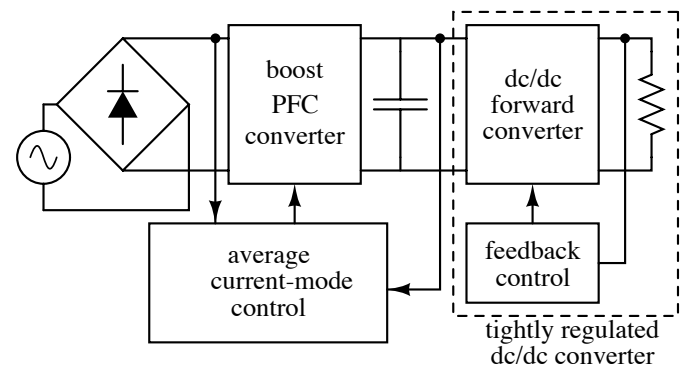

(a)

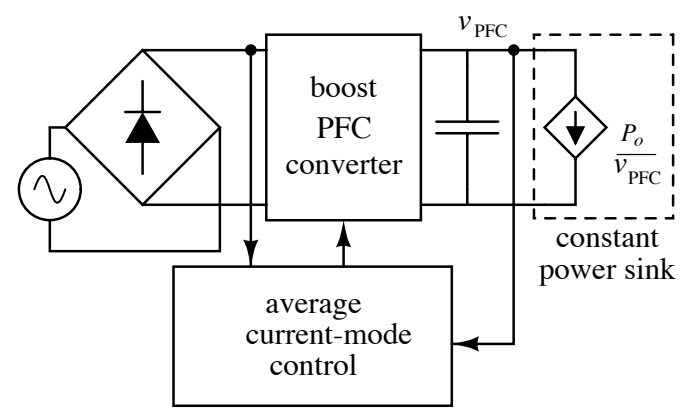

(b)

Fig. 1. Schematic of (a) the two-stage PFC power supply, and (b) the proposed model using a constant-power-sink termination.

voltage of the PFC boost stage), we observe that the downstream dc/dc converter maintains a well regulated DC output. In other words, its output power is constant under a fixed loading condition. The input power should also be constant on the average. Thus, if we consider low-frequency behavior, the downstream $\mathrm{dc} / \mathrm{dc}$ converter is simply a constant power sink, as shown in Fig. 1 (b). Suppose the load is fixed, and the power output is $P_{o}$. Then, the current drawn by the downstream converter can be expressed as

$$
i_{\mathrm{PFC}}(t)=\frac{P_{o}}{\eta v_{\mathrm{PFC}}(t)} \approx \frac{P_{o}}{v_{\mathrm{PFC}}(t)}
$$

where $v_{\mathrm{PFC}}$ is the output voltage of the PFC stage and $\eta$ is the efficiency of the $\mathrm{dc} / \mathrm{dc}$ converter. To simplify the calculation, we assume $\eta \approx 1$. Based on this simplified model, analysis of the converter's stability can be readily performed.

\section{Derivation OF Stability CRITERION}

\section{A. Generalized Averaged Model}

For the boost PFC stage, only CCM is considered here as it is commonly used for medium to high power applications. Average current-mode control is adopted for its better noise immunity and its popularity in industry. Fig. 2 shows the 


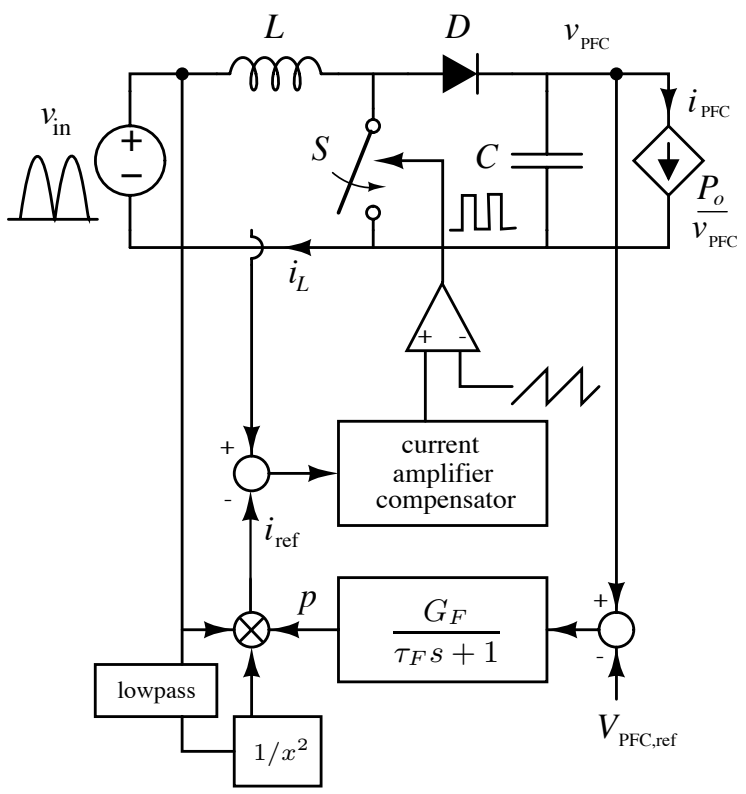

Fig. 2. Circuit schematic of the proposed model consisting of a boost PFC stage terminated by a constant power sink.

simplified schematic of our proposed model. The input current of the boost converter is forced to follow the sinusoidal input voltage envelope under average current-mode control. The "ideally shaped" input current waveform can be expressed as

$$
i_{\text {in }}(t)=\frac{p(t) \sqrt{2}}{V_{\text {in }, \mathrm{rms}}}\left|\sin \omega_{m} t\right|=\frac{p(t)}{V_{\mathrm{in}, \mathrm{rms}}^{2}} v_{\mathrm{in}}(t)
$$

where $p(t)$ is physically analogous to instantaneous power. By averaging over the switching period, the power stage can be modeled as

$$
\begin{aligned}
(1-d) v_{\mathrm{PFC}}(t) & =v_{\mathrm{in}}(t)-L \frac{d i_{\mathrm{in}}(t)}{d t} \\
(1-d) i_{\mathrm{in}}(t) & =C \frac{d v_{\mathrm{PFC}}(t)}{d t}+\frac{P_{o}}{v_{\mathrm{PFC}}(t)} .
\end{aligned}
$$

After combining (3) and (4) and eliminating $d$, we get

$$
\frac{C}{2} \frac{d v_{\mathrm{PFC}}(t)^{2}}{d t}=-P_{o}+i_{\text {in }}(t) v_{\text {in }}(t)-\frac{L}{2} \frac{d i_{\text {in }}(t)^{2}}{d t} .
$$

The power flowing through inductor $L$ is normally much smaller than that in capacitor $C$ operating near the line frequency, i.e., $\frac{C}{2} \frac{d v_{\mathrm{PFC}}(t)^{2}}{d t} \gg \frac{L}{2} \frac{d i_{\mathrm{in}}(t)^{2}}{d t}$. Thus, we have

$$
\frac{C}{2} \frac{d v_{\mathrm{PFC}}(t)^{2}}{d t}=-P_{o}+p(t)\left(1-\cos 2 \omega_{m} t\right)
$$

With a simple first-order filtering employed in the compensation, the feedback loop can be modeled as

$$
\frac{P(s)}{V_{\mathrm{PFC}}(s)}=\frac{-G_{F}}{\tau_{F} s+1}
$$

where $G_{F}$ is the overall dc gain and $\tau_{F}$ is the time constant of the voltage error amplifier. In the time domain, (7) is written as

$$
\tau_{F} \frac{d p(t)}{d t}+p(t)=-G_{F}\left(v_{\mathrm{PFC}}(t)-V_{\mathrm{PFC}, \mathrm{ref}}\right) .
$$

Thus, the system is described completely by (6) and (8).

\section{B. Fourier Series Expansion}

In this section, we will derive the loop gain corresponding to the line frequency, from which a stability criterion of the PFC boost stage can be derived. We begin with taking the Fourier series expansion of every state variable with the fundamental frequency being the line frequency, $\omega_{m}$. To simplify the calculation, we consider the first three terms, namely, the dc component, the fundamental frequency component at $\omega_{m}$, and the second harmonic component at $2 \omega_{m}$. In general, a variable $x(t)$ is expressed as

$$
x(t)=c_{o}+c_{1} e^{j \omega_{m} t}+\left[c_{1} e^{j \omega_{m} t}\right]^{*}+c_{2} e^{j 2 \omega_{m} t}+\left[c_{2} e^{j 2 \omega_{m} t}\right]^{*}
$$

where superscript $*$ denotes complex conjugation, and

$$
c_{n}=\frac{\omega_{m}}{2 \pi} \int_{t-\frac{2 \pi}{\omega_{m}}}^{t} x(\tau) e^{-j n \omega_{m} \tau} d \tau
$$

where $n=0,1$, and 2 . In the sequel, we will refer to this approximation as "second averaging" to distinguish it from the first averaging performed over the switching period. To avoid confusion due to possible mix-up of subscripting indices, we define $x_{n}$ as the $n$th harmonic coefficient of $v_{\mathrm{PFC}}(t)$, and $y_{n}$ as the $n$th harmonic coefficient of $p(t)$, with $n=0,1$ and 2 . Each coefficient is a complex number which can be expressed in Cartesian form as

$$
x_{n}=x_{n r}+j x_{n i} ; y_{n}=y_{n r}+j y_{n i}
$$

where $x_{n r}, y_{n r}$ refer to the real parts and $x_{n i}, y_{n i}$ refer to the imaginary parts.

Putting (10) in (6) results in three equations corresponding to the dc component, the fundamental frequency component and the second harmonic component:

$$
\begin{aligned}
& \frac{C}{2} \frac{d}{d t}\left(x_{0}^{2}+2 x_{1 r}^{2}+2 x_{1 i}^{2}+2 x_{2 r}^{2}+2 x_{2 i}^{2}\right)+P_{o} \\
= & y_{o}-y_{2 r} \\
& \frac{C}{2} \frac{d}{d t}\left(x_{0} x_{1}+x_{1 r} x_{2 r}+x_{1 i} x_{2 i}+j\left(x_{1 r} x_{2 i}-x_{1 i} x_{2 r}\right)\right) \\
& +\frac{j \omega_{m} C}{2}\left(x_{0} x_{1}+x_{1 r} x_{2 r}+x_{1 i} x_{2 i}\right. \\
& \left.+j\left(x_{1 r} x_{2 i}-x_{1 i} x_{2 r}\right)\right) \\
= & \frac{y_{1}}{2}-\frac{\left(y_{1}\right)^{*}}{4} \\
& \frac{C}{2} \frac{d}{d t}\left(2 x_{0} x_{2}+x_{1}^{2}\right)+j \omega_{m} C\left(2 x_{0} x_{2}+x_{1}^{2}\right) \\
= & y_{2}-\frac{y_{0}}{2} .
\end{aligned}
$$

Likewise, putting (10) in (8) results in

$$
\begin{aligned}
\tau_{F} \frac{d y_{0}}{d t}+y_{0} & =-G_{F}\left(x_{0}-V_{\mathrm{PFC}, \mathrm{ref}}\right)( \\
\tau_{F} \frac{d y_{1}}{d t}+\left(j \omega_{m} \tau_{F}+1\right) y_{1} & =-G_{F} x_{1} \\
\tau_{F} \frac{d y_{2}}{d t}+\left(j 2 \omega_{m} \tau_{F}+1\right) y_{2} & =-G_{F} x_{2}
\end{aligned}
$$




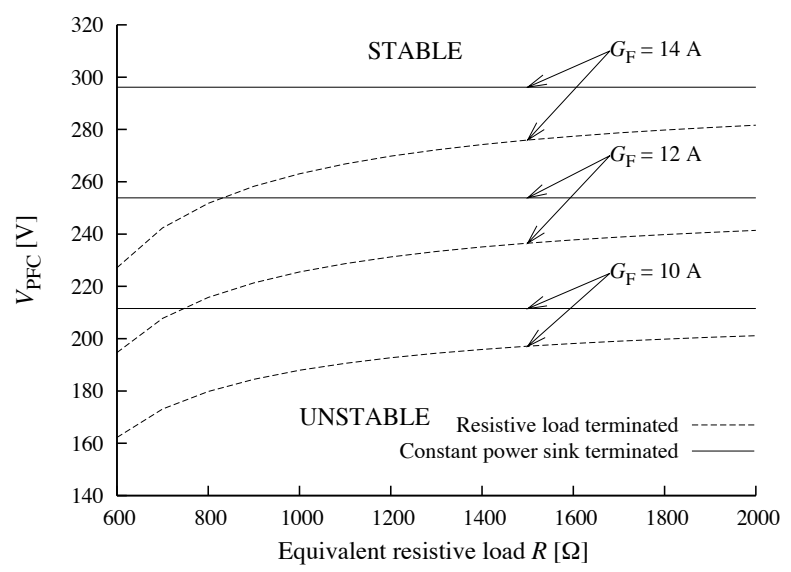

(a)

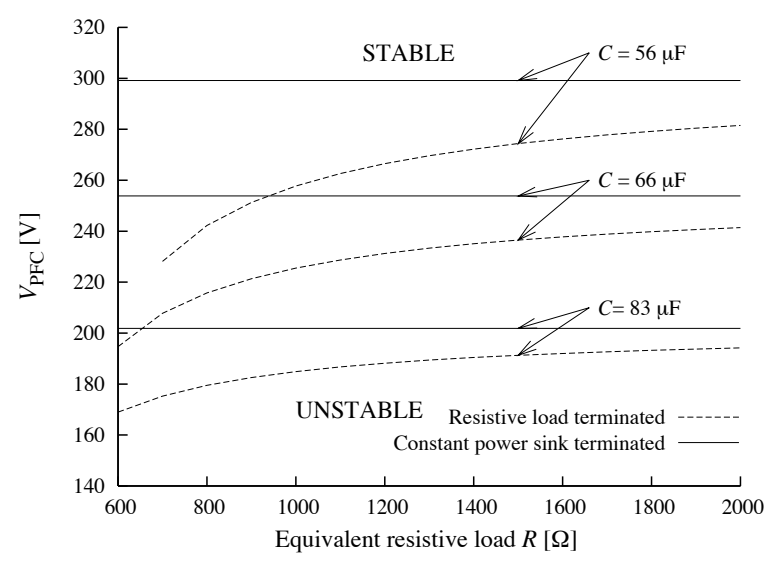

(b)

Fig. 3. Stability boundaries of the resistor terminated model and the proposed constant-power-sink terminated model. (a) $C=66 \mu \mathrm{F}, \tau_{\mathrm{F}}=9 \mathrm{~ms}$; (b) $G_{F}=12 \mathrm{~A}, \tau_{F}=9 \mathrm{~ms}$.

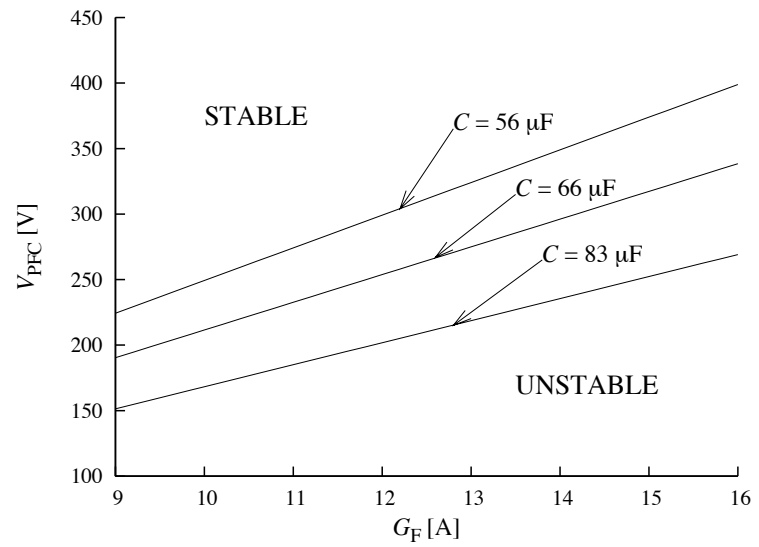

(a)

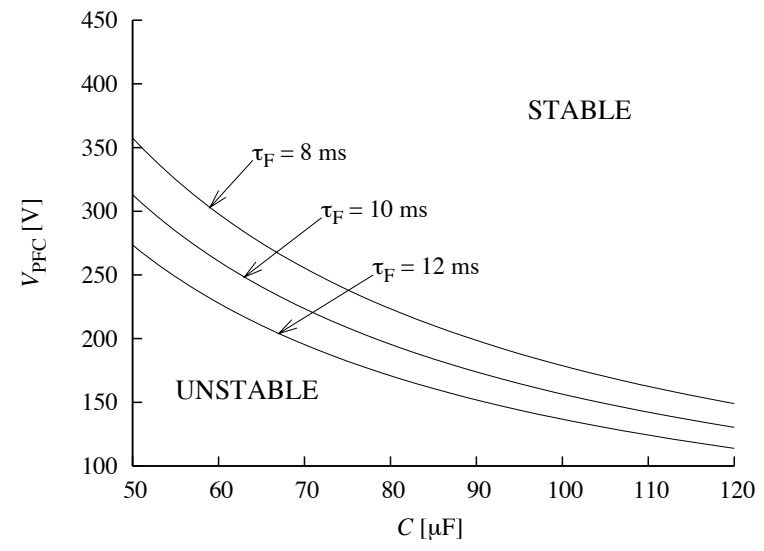

(b)

Fig. 4. Stability boundaries of the proposed constant-power-sink terminated model plotted in various parameter planes. (a) $G_{\mathrm{F}}-V_{\mathrm{PFC}}$ plane with $\tau_{F}=9$ ms; (b) $C-V_{\mathrm{PFC}}$ plane with $G_{F}=12$ A.

In the steady state, all time derivatives are zero. Thus, from (12), we obtain

$$
\begin{aligned}
{\left[\begin{array}{l}
x_{1 r} \\
x_{1 i}
\end{array}\right]=} & \frac{-1}{2 \omega_{m} C\left(x_{2 i}^{2}+x_{2 r}^{2}-x_{o}^{2}\right)} \\
& {\left[\begin{array}{cc}
x_{2 i} & 3 x_{o}-3 x_{2 r} \\
-x_{o}-x_{2 r} & 3 x_{2 i}
\end{array}\right]\left[\begin{array}{l}
y_{1 r} \\
y_{1 i}
\end{array}\right] }
\end{aligned}
$$

Similarly, enforcing the steady-state condition to (15), we have

$$
\begin{aligned}
{\left[\begin{array}{l}
y_{1 r} \\
y_{1 i}
\end{array}\right]=} & \frac{-1}{1+\tau_{F}^{2} \omega_{m}^{2}} \\
& {\left[\begin{array}{cc}
G_{F} & G_{F} \tau_{F} \omega_{m} \\
-G_{F} \tau_{F} \omega_{m} & G_{F}
\end{array}\right]\left[\begin{array}{c}
x_{1 r} \\
x_{1 i}
\end{array}\right] }
\end{aligned}
$$

From (17) and (18), the round-trip signal transfer function $\mathbf{M}$ is

$$
\begin{aligned}
\mathbf{M}= & \frac{-1}{1+\tau_{F}^{2} \omega_{m}^{2}}\left[\begin{array}{cc}
G_{F} & G_{F} \tau_{F} \omega_{m} \\
-G_{F} \tau_{F} \omega_{m} & G_{F}
\end{array}\right] \\
& \cdot \frac{-1 / 2 \omega_{m} C}{\left(x_{2 i}^{2}+x_{2 r}^{2}-x_{o}^{2}\right)}\left[\begin{array}{cc}
x_{2 i} & 3 x_{o}-3 x_{2 r} \\
-x_{o}-x_{2 r} & 3 x_{2 i}
\end{array}\right] .
\end{aligned}
$$

With the assumption of $x_{o}=V_{\mathrm{PFC}, \text { ref }}$, all the second harmonic components become zero. Thus, (19) can be further simplified as

$$
\mathbf{M}=\frac{G_{F}}{2 x_{o} \omega_{m} C\left(1+\tau_{F}^{2} \omega_{m}^{2}\right)}\left[\begin{array}{cc}
\tau_{F} \omega_{m} & -3 \\
1 & 3 \tau_{F} \omega_{m}
\end{array}\right] .
$$

The magnitude of the total loop gain $\left|T_{x}\right|$ is obtained from the eigenvalue of the matrix $\mathbf{M}$, i.e.,

$$
\left|T_{x}\right|=\frac{2 G_{F} \tau_{F} \omega_{m}+G_{F} \sqrt{-3+\tau_{F}^{2} \omega_{m}^{2}}}{2 \omega_{m} x_{o} C\left(1+\tau_{F}^{2} \omega_{m}^{2}\right)} .
$$

For stable operation, $\left|T_{x}\right|<1$. Thus, the stability criterion of the PFC stage terminated with a constant power sink, which effectively represents the two-stage PFC power supply, is given by

$$
x_{o}>\frac{G_{F}\left(2 \tau_{F} \omega_{m}+\sqrt{-3+\tau_{F}^{2} \omega_{m}^{2}}\right)}{2 \omega_{m} C\left(1+\tau_{F}^{2} \omega_{m}^{2}\right)},
$$

which differs significantly from the stability criterion derived in [2] for the PFC boost converter terminated with a resistive 


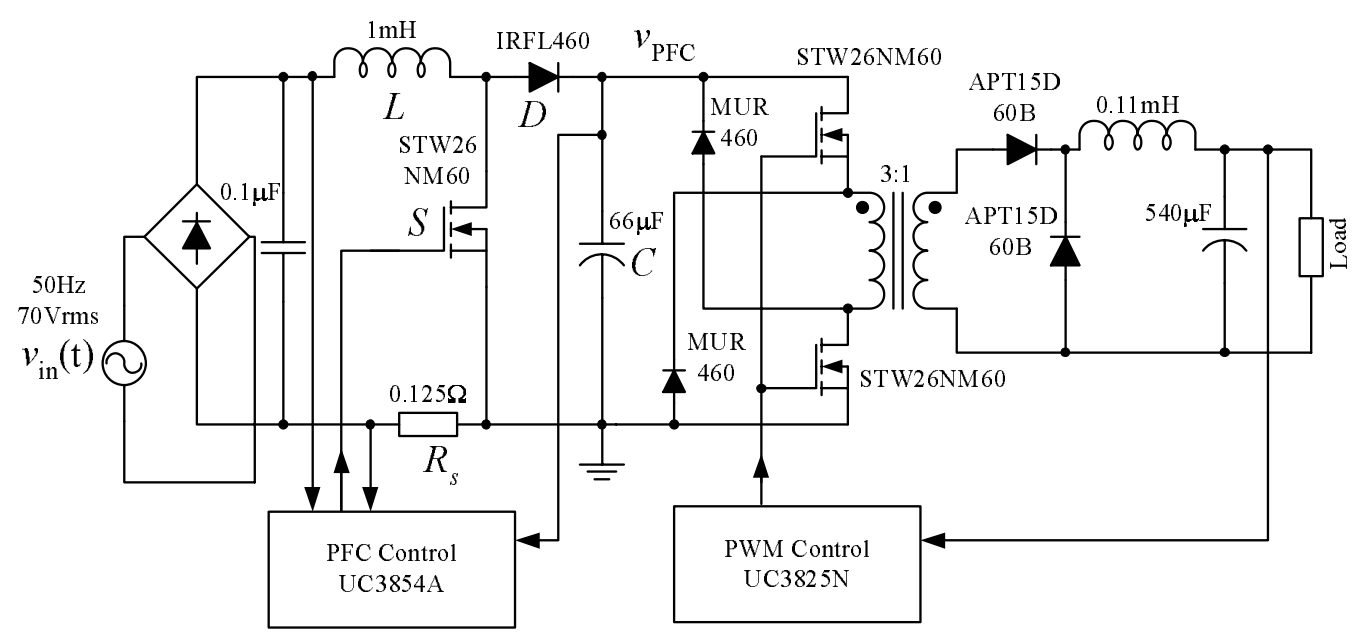

Fig. 5. Experimental circuit of the complete two-stage PFC power supply.

load. For convenience of comparison, we reproduce the stability criterion for the PFC boost converter terminated with a resistive load as given in (23) at the bottom of this page [2]. By inspecting (22) and (23), both criteria reveal that the line-frequency stability is dependent upon $G_{F}, \tau_{F}, C$ and $x_{o}$. However, for the resistor terminated PFC boost converter, the load resistance $R$ or equivalently the output power affects the stability, whereas for the constant-power-sink terminated PFC boost converter, the output power does not play a role in determining the stability.

\section{Design-Oriented Stability Boundaries}

In this section, we present (22) and (23) graphically with $\mathrm{y}$-axis being $V_{\mathrm{PFC}}$ and $\mathrm{x}$-axis being a particular choice of parameter. Then, the stability boundaries clearly show the lower limits of $V_{\mathrm{PFC}}$ below which the system will become unstable and exhibit oscillation at the line frequency.

Fig. 3 shows the stability boundaries plotted on the $R-V_{\mathrm{PFC}}$ plane with different parameters and compares the two models of the resistor terminated PFC boost converter and constantpower-sink terminated PFC boost converter. From the results, we generally observe that the constant-power-sink terminated PFC boost converter has a more restricted stable region. To probe further, Fig. 4 shows the stability boundaries on a few other parameter planes.

\section{Experimental Measurements}

A two-stage PFC power supply has been constructed for verification purpose, with the schematic shown in Fig. 5. The PFC pre-regulator is a non-isolated boost converter under average current-mode control accomplished by UC3854A PFC controller, as detailed in Fig. 6. The downstream converter is a

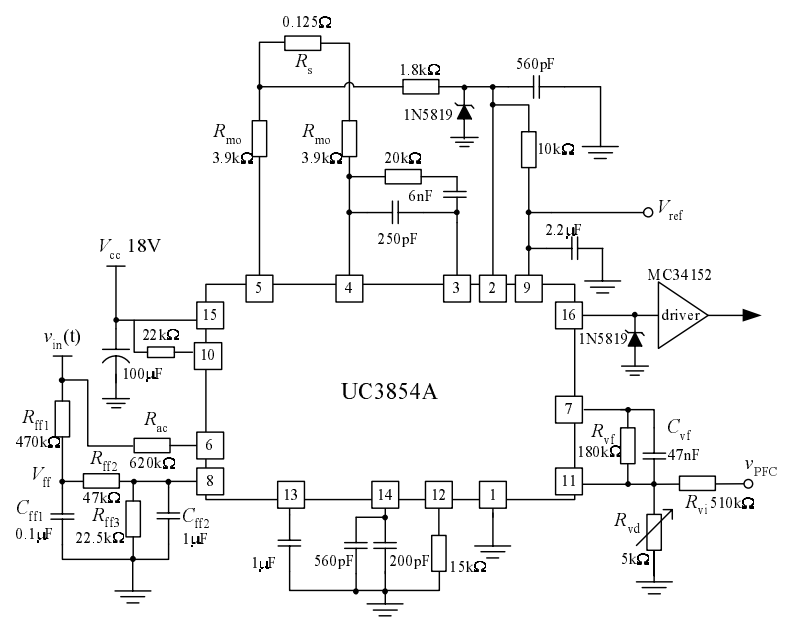

Fig. 6. Detailed schematic of PFC controller circuit.

double-wheeler forward converter using voltage-mode control accomplished by standard UC3825N PWM controller. The key circuit parameters are shown in Table I.

\section{A. Comparison of Resistor Terminated and Constant-Power- Sink Terminated PFC Converters}

When line-frequency instability occurs, "period-doubling" waveforms can be observed in the input current and the output voltage, as shown in Fig. 7 . It degrades the PFC converter performance. Specifically, the power factor decreases, the output ripple voltage increases, and the peak input current also increases. Fig. 8 presents the deterioration of these performance parameters as the system goes from the stable region to deep unstable region. We also compare the conventional

$$
x_{o}>\frac{G_{F} R\left[\omega_{m}^{2} C R \tau_{F}-2+\sqrt{1-4 \omega_{m}^{2} C R \tau_{F}-3 \omega_{m}^{2} \tau_{F}^{2}+\frac{1}{4} \omega_{m}^{2} C^{2} R^{2}\left(\omega_{m}^{2} \tau_{F}^{2}-3\right)}\right]}{\left(4+\omega_{m}^{2} C^{2} R^{2}\right)\left(1+\omega_{m}^{2} \tau_{F}^{2}\right)} .
$$




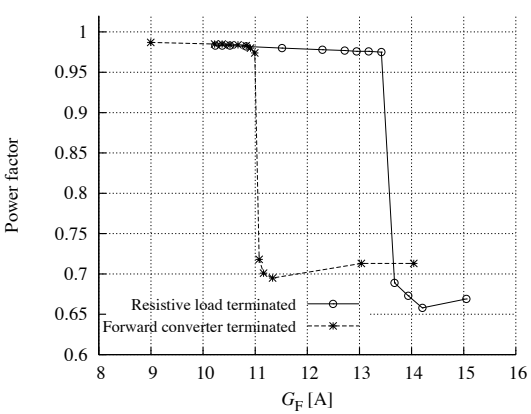

(a)

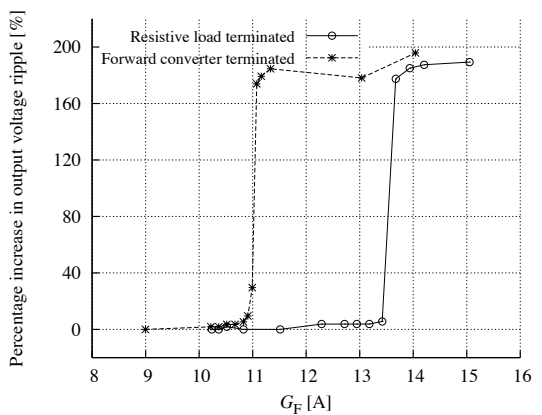

(b)

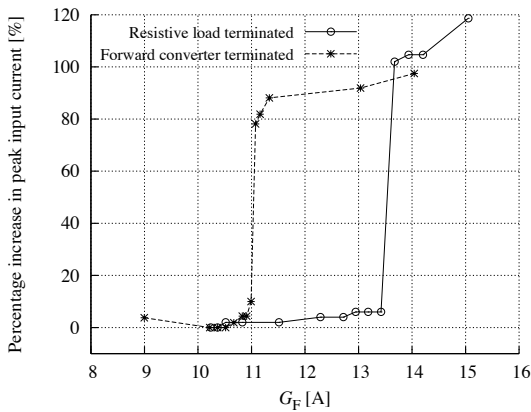

(c)

Fig. 8. Performance degradation due to line-frequency instability. (a) Power factor; (b) output ripple voltage; and (c) peak input current.

TABLE I

CIRCUIT PARAMETERS USED IN EXPERIMENTS

\begin{tabular}{ll}
\hline Parameters of PFC stage & Values \\
\hline Rectified line voltage $V_{\text {in,rms }}$ & $70 \mathrm{Vrms}, 50 \mathrm{~Hz}$ \\
PFC stage reference output voltage $V_{\mathrm{PFC}, \mathrm{ref}}$ & $240 \mathrm{~V}$ \\
Switching frequency $f_{s w 1}$ & $100 \mathrm{kHz}$ \\
Inductance $L$ & $1 \mathrm{mH}$ \\
Output capacitance $C$ & $66 \mu \mathrm{F}$ \\
Nominal voltage feedback gain $G_{F}$ & $11.6 \mathrm{~A}$ \\
Nominal feedback time constant $\tau_{F}$ & $8.6 \mathrm{~ms}$ \\
Forward converter reference output $V_{o, \text { ref }}$ & $24 \mathrm{~V}$ \\
Forward converter switching frequency $f_{s w 2}$ & $100 \mathrm{kHz}$ \\
\hline
\end{tabular}

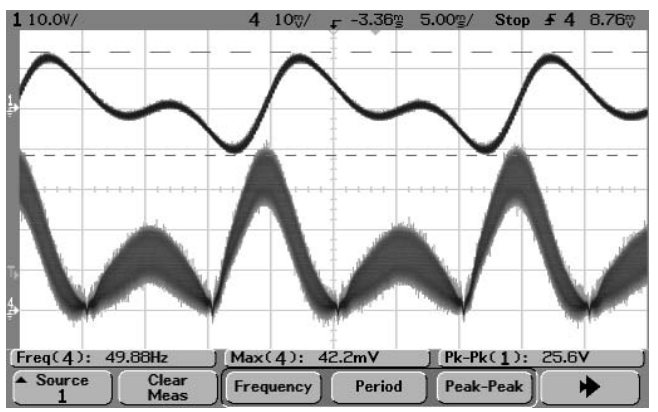

Fig. 7. Measured waveform showing line-frequency instability in the complete two-stage system. Upper trace is output ripple voltage $(10 \mathrm{~V} / \mathrm{div})$; lower trace of left panel is rectified input current $(500 \mathrm{~mA} / \mathrm{div})$.

resistor terminated PFC converter and the complete two-stage PFC power supply. For the complete two-stage system, the degradation begins at a much lower value of $G_{F}$ than the resistor terminated system. This once again verifies that the two-stage system is more prone to line-frequency instability.

\section{B. Verification of the Stability Boundaries}

In this subsection, the stability boundaries derived in Section III are verified experimentally. Measured data are plotted along with the analytical results to facilitate comparison, as shown in Figs. 9 and 10. The measured data generally match well with the analytical results. The small discrepancies can be attributed to the errors introduced due to the omission of higher order harmonics in our analysis. Besides, the assumption of $x_{o}=V_{\mathrm{PFC}, \text { ref }}$ incurs a small DC offset. In the practical circuit, the internal multiplier gain varies slightly as the load changes, resulting in error in the loop gain calculation. The measured results are therefore expected to deviate slightly from the analytical results.

\section{AppliCATIONS}

In this section, we attempt to apply the stability criterion derived to the design of the voltage feedback loop in PFC converters. We will develop some guidelines for ensuring stable operation of PFC converters.

In order to improve the transient performance, input current distortion is the usual trade-off considered [4], [5]. In fact, when the voltage loop gain is increased to a certain level, the converter may become unstable. Thus, stability need to be thoroughly evaluated during the design process. For a quick reference, normalized stability boundaries in Fig. 11 can be used to predict the PFC converter's stability. For comparison, $G_{F}$ and $\tau_{F}$ can be mapped to the actual circuit parameters by

$$
\begin{aligned}
G_{F} & =\frac{R_{\mathrm{vf}}}{R_{\mathrm{vi}}} \frac{R_{\mathrm{mo}}}{R_{\mathrm{s}} R_{\mathrm{ac}}}\left(\frac{V_{\mathrm{in}, \mathrm{rms}}}{V_{\mathrm{ff}}}\right)^{2} K \\
\tau_{F} & =R_{\mathrm{vf}} C_{\mathrm{vf}}
\end{aligned}
$$

where all component variables mentioned above are shown in Fig. 6, i.e., $R_{\mathrm{vf}}$ and $R_{\mathrm{vi}}$ are the feedback resistor and input resistor of the voltage error amplifier respectively; $C_{\mathrm{vf}}$ is the feedback capacitor; $R_{\mathrm{mo}}$ is the input resistor of the current amplifier; $R_{\mathrm{s}}$ is the current sensing resistor; $R_{\mathrm{ac}}$ is the resistor connecting the input voltage to the multiplier; $V_{\mathrm{ff}}$ is the feedforward voltage; and $K$ is the internal gain of the multiplier which has been found experimentally to be $1.12 \mathrm{~V}$.

To improve stability, an effective way is to increase the output capacitor value, the price to pay being the higher component cost and extra space. Besides, the overall dc gain and time constant can be adjusted at the expense of degraded transient and regulation performances. In some cases where the output voltage of the pre-regulator stage is not tightly specified, the reference output voltage can be increased to improve stability. Table II summarizes the tradeoffs for stability improvement. 
TABLE II

SUMMARY OF PERFORMANCE TRADEOFFS FOR STABILITY IMPROVEMENT

\begin{tabular}{|l|l|l|l|l|}
\hline Parameters & $\begin{array}{l}\text { Adjusting } \\
\text { components }\end{array}$ & $\begin{array}{l}\text { Increase/decrease for } \\
\text { stability improvement }\end{array}$ & Other benefits & Performance tradeoffs \\
\hline \hline$C$ & $C$ & Increase & $\begin{array}{l}\text { Smaller output ripple } \\
\text { Lower current distortion } \\
\text { Smaller Transient overshoot } \\
\text { Longer holdup time }\end{array}$ & $\begin{array}{l}\text { Cost } \\
\text { Space }\end{array}$ \\
& & & Lower current distortion & Regulation \\
& & & Transient response \\
\hline$G_{F}$ & $\begin{array}{l}R_{\mathrm{vf}}, R_{\mathrm{vi}}, R_{\mathrm{mo}}, R_{\mathrm{s}}, R_{\mathrm{ac}}, \\
R_{\mathrm{ff} 1}, R_{\mathrm{ff} 2}, R_{\mathrm{ff} 3}, C_{\mathrm{ff} 1}, C_{\mathrm{ff} 2}\end{array}$ & Decrease & Lower current distortion & Transient response \\
\hline$\tau_{F}$ & $C_{\mathrm{vf}}, R_{\mathrm{vf}}$ & Increase & Longer holdup time & Voltage stress \\
\hline$V_{\mathrm{PFC}}$ & $R_{\mathrm{vi}}, R_{\mathrm{vd}}$ & Increase & \\
\hline
\end{tabular}

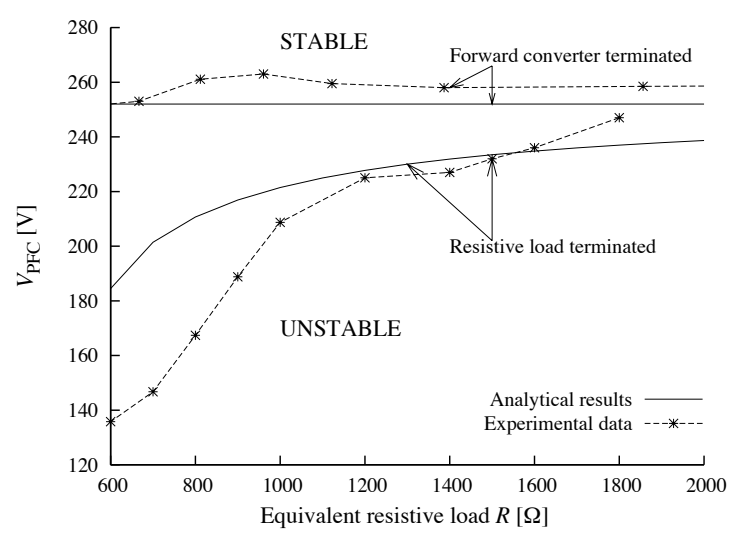

Fig. 9. Experimental verification and comparison of stability boundaries between the resistor terminated model and the complete two-stage system. $G_{F}=11.6 \mathrm{~A}, C=66 \mu \mathrm{F}, \tau_{F}=8.6 \mathrm{~ms}$.

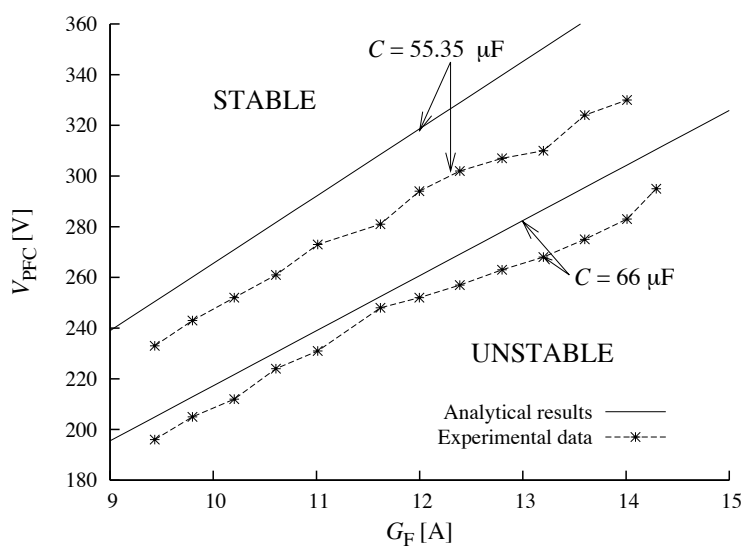

Fig. 10. Experimental verification of stability boundaries of the constantpower-sink terminated model in $G_{F}-v_{\mathrm{PFC}}$ plane with $\tau_{F}=8.6 \mathrm{~ms}$.

\section{CONCLUSION}

In this paper, the low-frequency instability in boost PFC converters is examined in the light of sustained oscillation at the line frequency. A simple model of a two-stage PFC power supply has been proposed. Essentially the downstream $\mathrm{dc} / \mathrm{dc}$ converter is modeled as a constant power sink cascaded with the PFC boost stage. Using on this model, we derived the closed-form loop gain of the line frequency component and succeeds in capturing the stability information in terms

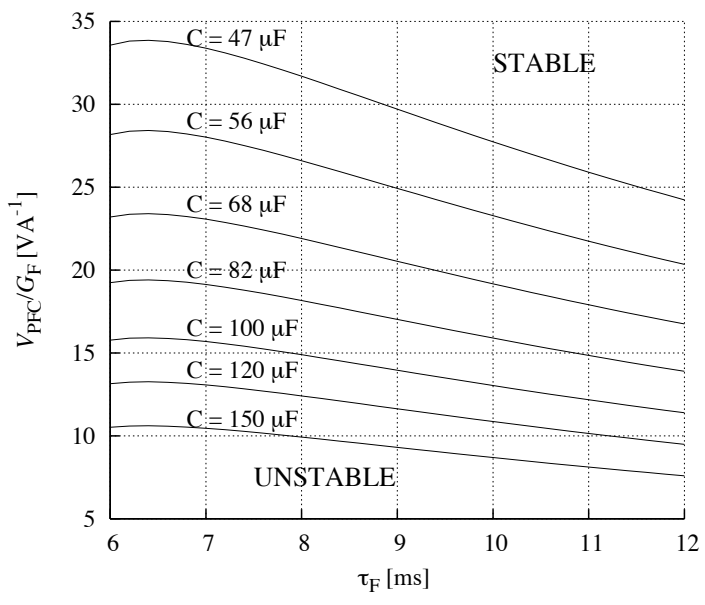

Fig. 11. Design aid to verify stability of PFC power supply.

of a closed-form stability condition, which can be visualized graphically in terms of stability boundaries. Stable regions and unstable regions are identified and compared between the resistor terminated model and the proposed model. Our results show that the two-stage PFC power supply has a more restricted stability region and is more prone to line-frequency instability. The stability boundaries have been verified experimentally. Finally, the derived stability criterion can be used to generate design guidelines for stable operation.

\section{ACKNOWLEDGMENT}

This work was supported by Hong Kong Polytechnic University under Research Grant G-U284.

\section{REFERENCES}

[1] M. Orabi and T. Ninomiya, "Nonlinear dynamics of power-factorcorrection converter," IEEE Trans. Ind. Electron., vol. 50, no. 6, pp. 1116-1125, Dec. 2003.

[2] S. C. Wong, C. K. Tse, M. Orabi, and T. Ninomiya, "The method of double averaging: an approach for modeling power-factor-correction power converters," IEEE Trans. Circuits and Systems I, vol. 53, no. 2, pp. 454-462, Feb. 2006.

[3] O. Dranga, G. Chu, C. K. Tse, and S. C. Wong, "Stability analysis of two-stage PFC Power Supplies," IEEE Power Electron. Specialists Record, Jeju, Korea, pp. 594-598, June 2006.

[4] P. C. Todd, "UC3854 controlled power factor correction circuit design," Application Note U-134, Unitrode, 2003.

[5] A. Fernandez, J. Sebastian, and P. Villegas, "Dynamic limits of a powerfactor preregulator," IEEE Trans. Ind. Electron., vol. 52, no. 1, pp. 7786, Feb. 2005. 